\title{
Can lichen sclerosus be diagnosed by preputial appearance or symptoms?
}

This article was published in the following Dove Press journal: Research and Reports in Urology

\author{
Taiki Kato' \\ Kentaro Mizuno ${ }^{2}$ \\ Hidenori Nishio ${ }^{2}$ \\ Yoshinobu Moritoki' \\ Akihiro Nakane' \\ Satoshi Kurokawa' \\ Hideyuki Kamisawa' \\ Tetsuji Maruyama ${ }^{3}$ \\ Takahiro Yasui' \\ Yutaro Hayashi ${ }^{2}$ \\ 'Department of Nephro-urology, \\ Nagoya City University Graduate \\ School of Medical Sciences, Kawasumi, \\ Mizuho-cho, Mizuho-ku, Nagoya, Japan; \\ ${ }^{2}$ Department of Pediatric Urology, \\ Nagoya City University Graduate \\ School of Medical Sciences, Kawasumi, \\ Mizuho-cho, Mizuho-ku, Nagoya, Japan; \\ ${ }^{3}$ Department of Urology, Nagoya \\ City East Medical Center, Wakamizu, \\ Chikusa-ku, Nagoya, Japan
}

Correspondence: Kentaro Mizuno Department of Pediatric Urology, Nagoya City University Graduate School of Medical Sciences, I Kawasumi, Mizuhocho, Mizuho-ku, Nagoya 467-860I, Japan Tel +8I 528538266

Fax +8I 528523179

Email kmizuno@med.nagoya-cu.ac.jp
Introduction: Lichen sclerosus (LS), or balanitis xerotica obliterans, is a chronic, inflammatory disease accompanied by cicatrizing skin conditions resulting in pathologic phimosis. LS can be detected clinically by the whitish appearance of the glans or the foreskin, thickened, nonretractable foreskin, dysuria, and spraying. However, diagnosis is confirmed histopathologically. Purpose: The aim of this study was to investigate whether LS can be diagnosed on the basis of clinical manifestation and symptoms.

Patients and methods: A retrospective analysis was performed, which included boys referred to our institutions for phimosis evaluation between May 2001 and December 2016. Pediatric urologists clinically diagnosed LS preoperatively. Boys with voiding problems, recurrent balanoposthitis, or who were unresponsive to topical steroid treatment underwent surgical treatment. Patients who underwent religious circumcisions were not included.

Results: Three-hundred twenty boys diagnosed with phimosis were identified, and 71 (22\%) boys underwent surgery. Fifty-eight boys underwent complete or partial circumcision, and 13 boys underwent a dorsal slit or preputioplasty. Thirty foreskin samples were sent for histopathologic examination. Histopathologic evaluation showed evidence of LS in 13 of 30 (43\%) circumcised boys, chronic inflammation in ten (33\%), no abnormality in four (13\%), dysplasia in two (7\%), and acute inflammation in one ( $3 \%$ ). In the 22 boys who were clinically diagnosed with LS, 13 patients had histopathologic evidence of LS (59\%). Visually, eleven cases had whitish foreskins and 21 cases had thickened nonretractable foreskins, of which there were seven $(64 \%)(P>0.05)$ and $12(57 \%)(P<0.01)$ cases, respectively. These cases were histopathologically diagnosed with LS. Both whitish and thickened, nonretractable foreskin was observed in eight patients, of which seven $(88 \%)(P<0.01)$ were also histologically diagnosed with LS. There was no correlation between histopathologic diagnosis and preoperative symptoms such as dysuria, ballooning, spraying, or penile pain $(P>0.2)$.

Conclusion: It is difficult to reliably distinguish whether a patient has histopathological LS by clinical findings alone.

Keywords: balanitis xerotica obliterans, lichen sclerosus, circumcision, histology, child

\section{Introduction}

Lichen sclerosus (LS) is a chronic, progressive, inflammatory lymphocyte-mediated dermatologic disease that affects the anogenital area in women and the prepuce and glans in men, excluding the perianal region. ${ }^{1}$ The clinical features of LS include porcelain-white discoloration of the entire foreskin margin and thickened, sclerotic, and nonretractable foreskin. The recommended treatment for LS is complete circumcision $^{2}$ because partial circumcision is followed by recurrent disease in $50 \%$ of cases. ${ }^{3}$ 
Circumcision is usually curative, although some patients may require further treatment for meatal stenosis or urethral stricture. $^{4}$

LS can be detected clinically; however, the diagnosis is based on histopathologic examinations. Bochove-Overgaauw et $\mathrm{al}^{5}$ reported that clinical findings underestimated the incidence of LS by $\sim 50 \%$ when compared to biopsies of circumcised foreskin in children. We hypothesized that if useful clinical findings for diagnosing LS were clarified, then the recurrence rate could be reduced by performing complete circumcision instead of partial circumcision. The aim of this retrospective study was to use histologic evaluation to determine whether a correct diagnosis of LS can be made by clinical evaluation.

\section{Patients and methods}

We carried out a retrospective analysis of the clinical and pathologic findings of LS at our institutions. Between May 2001 and December 2016, a total of 418 patients with a mean age of 5 years (range 2-20 years) were referred for evaluation of phimosis and were considered for possible surgery. Absolute indications for surgery were pathologic phimosis associated with voiding problems, recurrent balanoposthitis, or unresponsiveness to topical steroid treatment. Resected foreskin samples were sent for histopathologic examination and were assessed by experienced pathologists in our pathology department. A preoperative clinical diagnosis of LS was made by experienced pediatric urologists based on the appearance of white discoloration at the tip of the prepuce or a ring of thickened tissue with extensive scarring and nonretractable foreskin. The presence of whitish foreskin, scarring, and nonretractable foreskin was determined by descriptions or photographs in the medical records. The symptoms of dysuria, pain, and incontinence were based on the subjective judgment of the patient or the parents. Urinary conditions, such as ballooning and spraying, were determined by the physicians during urination. Patients who underwent religious or cultural circumcision were not included.

Fisher's exact test was used to evaluate the statistical significance between patients with and without histopathologic LS. Data were considered statistically significant if $P<0.05$. The statistical analyses were made using SPSS statistics software, version 22 (IBM Corp., Armonk, NY, USA). The research procedure was approved (No. 60160221) by the institutional review board (IRB) of Nagoya City University Graduate School of Medical Sciences (Nagoya, Japan). Patient's informed consent to review their medical record was not required by the IRB. We disclosed the information of implementation status of this research and ensured opportunities for refusal on our hospital website and inside our hospital.

\section{Results}

A total of 418 patients were identified, of whom 98 were excluded from the study for various reasons (including paraphimosis, buried penis, and balanoposthitis at presentation). Of the remaining 320 boys, 171 (53.4\%) required no therapy, $78(24 \%)$ were treated with topical steroids, and $71(22 \%)$ underwent surgery. Fifty-eight boys underwent complete circumcision or partial circumcision, and 13 boys underwent dorsal slit or triple incision preputioplasty. Thirty foreskins sampled from 58 boys who underwent complete circumcision or partial circumcision were evaluated histopathologically. Of 58 boys, 30 foreskin samples were available for histopathologic examination with consent from their parents. Three boys were treated with topical steroids for 2 weeks to 6 months preoperatively and all the boys discontinued topical steroid treatment for $>1$ month before surgery. Twenty-four boys underwent complete circumcision, and six boys underwent preputioplasty and partial circumcision.

The mean age of the patients with histopathologic LS was 8.3 years, and the median age was 8.8 years (Figure 1). Histopathologic evaluation showed that LS was found in 13 of 30 foreskin samples (43\%). Ten of 30 samples (33\%) had a histopathologic diagnosis of chronic inflammation, four of 30 samples (13\%) had no abnormalities, two were diagnosed as dysplasia, and one was diagnosed with acute inflammation. Of the 22 boys who were clinically diagnosed with LS, 13 boys had histopathologic evidence of LS (Figure 2). Thus, nine boys were clinically diagnosed with LS; however, they had no histopathologic evidence of LS. In our study, clinical suspicion of LS was unreliable, with a diagnostic accuracy as low as $59 \%$.

The preoperative preputial appearance and symptoms are summarized in Table 1. Visually, eleven cases had whitish foreskins, of which seven (64\%) were histopathologically diagnosed with LS $(P=0.08)$. Twenty-one cases had thickened, nonretractable foreskins, of which 12 (57\%) cases were histopathologically diagnosed with LS $(P<0.01)$. Both whitish and thickened, nonretractable foreskin were observed in eight patients, of which seven (88\%) were also histologically diagnosed with LS $(P<0.01)$. On the other hand, of the ten boys who were histopathologically diagnosed with chronic inflammation, six boys were clinically diagnosed with LS and four boys did not have LS. One boy had whitish foreskin, six boys had thickened, nonretractable foreskins, one boy had both whitish and thickened, and one boy was without both. 
Number of patients

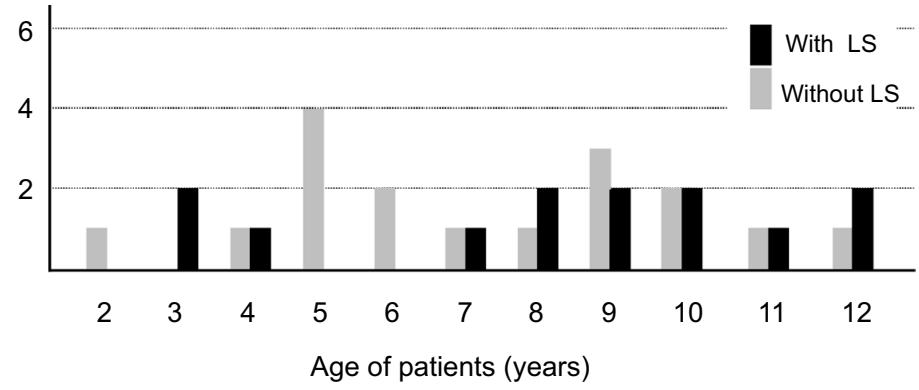

Figure I Age distribution of boys with LS (black bar) and without LS (gray bar). Abbreviation: LS, lichen sclerosus.

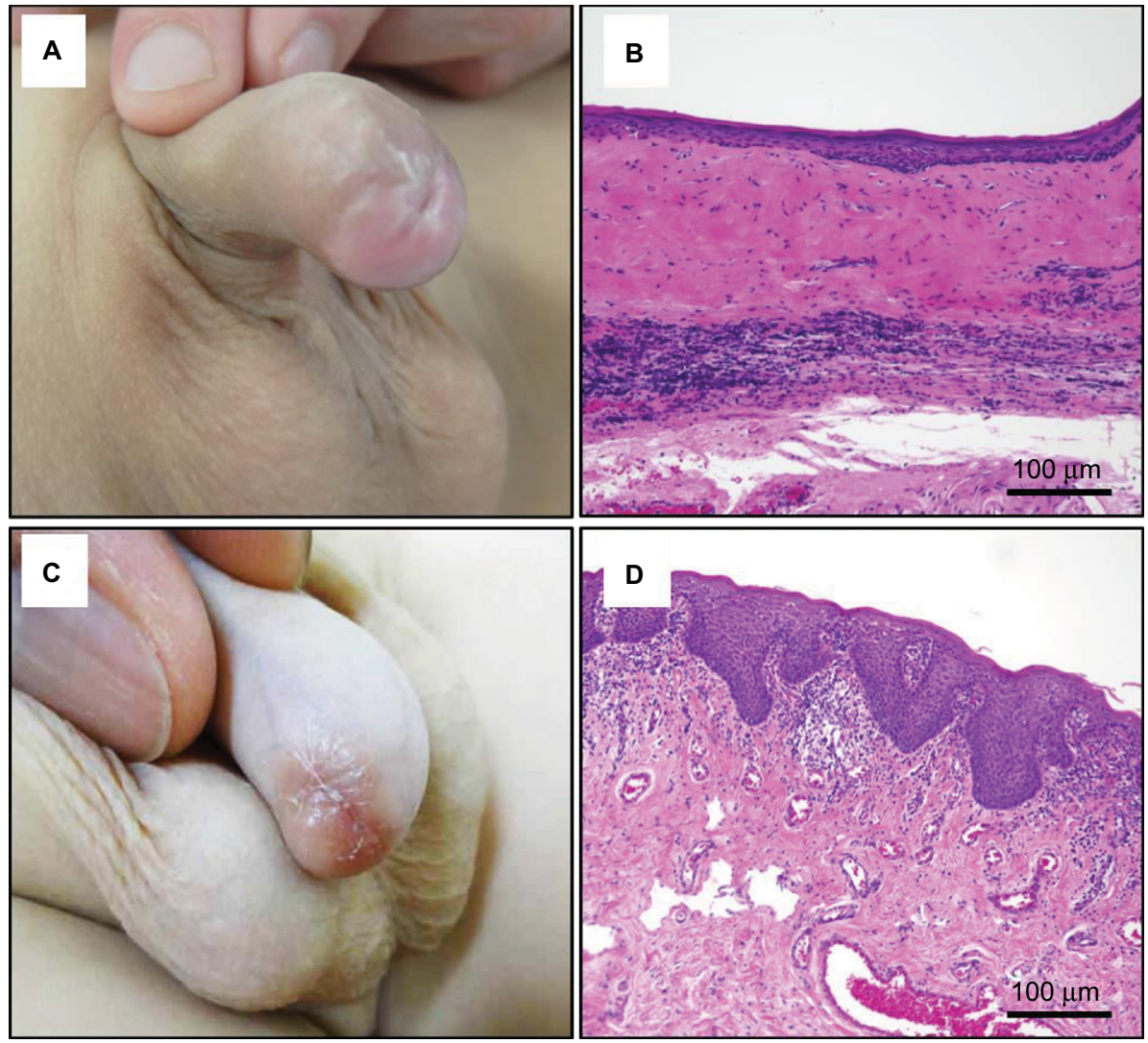

Figure 2 Preputial appearance and histopathologic findings of LS (A,B) and chronic inflammation (C,D).

Notes: (A) Pathologic phimosis, whitish discoloration, and thickened, nonretractable foreskin suggesting LS. (B) Histology shows that there is hyperkeratosis with atrophic epidermis, a wide homogenous zone with the basal part of band-like inflammatory infiltration, diagnosed as LS. Magnification $\times 100$. (C) The boy was clinically diagnosed with LS as (A). (D) Inflammatory cell infiltration diffusely occurs in the epidermis and dermis. There was no hyperkeratosis or liquefaction degeneration diagnosed as chronic inflammation. Magnification $\times 100$.

Abbreviation: LS, lichen sclerosus.

The clinical symptoms were dysuria in $10 \%(3 / 30)$, history of balanitis in $37 \%(11 / 30)$, spraying in $20 \%(6 / 30)$, and ballooning of the foreskin on micturition in 50\% (15/30). There was no correlation between preoperative symptoms and histopathologic diagnosis.
The mean follow-up period was 15 months (range: 1-96 months). One boy with histopathologic LS had a narrowed meatal lumen and underwent meatoplasty at the same time as circumcision. Another boy with histopathologic LS went on to require dilation for meatal stenosis 4 months postoperatively. 
Table I Clinical features of patients with and without histologic LS

\begin{tabular}{llll}
\hline & $\begin{array}{l}\text { With LS } \\
(\mathbf{N}=13)\end{array}$ & $\begin{array}{l}\text { Without } \\
\text { LS (N=17) }\end{array}$ & P-value \\
\hline Preoperative appearance & 7 & 4 & 0.081 \\
Whitish foreskin & 12 & 9 & $<0.01$ \\
Thickened nonretractable foreskin & 1 & $\mathrm{I}$ & $<0.01$ \\
Both & 7 & & \\
Preoperative symptoms & $\mathrm{I}$ & 2 & 0.603 \\
Dysuria & 6 & 9 & 0.354 \\
Ballooning & 4 & 2 & 0.204 \\
Spraying & $\mathrm{I}$ & 4 & 0.261 \\
Pain during urination & $\mathrm{I}$ & 2 & 0.603 \\
Penile pain & 0 & $\mathrm{I}$ & 0.567 \\
Incontinence & 0 & $\mathrm{I}$ & 0.567 \\
Penile itching & 2 & 9 & 0.397 \\
History of balanoposthitis & & & \\
\hline
\end{tabular}

Abbreviation: LS, Lichen sclerosus.

Neither of the two patients required further intervention. No postoperative complications were noted in the other boys.

\section{Discussion}

LS is a chronic, inflammatory disorder presenting as a sclerosing, atrophy of the glans and foreskin, leading to meatal stenosis and acquired phimosis. ${ }^{4}$ LS appears to be lymphocyte-mediated, and an autoimmune etiology is likely, considering its strong association with autoimmune disorders (eg, autoimmune thyroid disease). ${ }^{6,7}$ It was recently reported that an LS urethral stricture is associated with chronic systemic diseases, including hypertension, hyperlipidemia, or diabetes in adult men. However, the cause of LS has not been identified.

LS was thought to be a disease that occurred only in adults until a pediatric case was published in $1962 .{ }^{8}$ In 1999 , the incidence of LS was estimated to be 0.3 cases $/ 1,000$ boys per year, and $0.5 \%$ of boys had the disease by the age of $15 .{ }^{9} \mathrm{In}$ recent years, its incidence appears to be increasing in children because more foreskin samples may have been submitted to histopathologic examination. ${ }^{4}$ In our study, accurate differential diagnoses of LS and chronic inflammation were difficult to determine through clinical appearance alone, and we may underestimate LS if histopathologic examination is not performed. Celis et a ${ }^{10}$ reported that the global incidence of LS is 35\% among circumcised children younger than 18 years. LS was previously thought to occur only in school-aged boys ${ }^{11}$ although it was recently reported that LS can occur even in boys under 5 years. ${ }^{4}$ In our study, histologic LS was confirmed in two patients aged 3 and 4 years.
Initial LS lesions are white, polygonal, flat-topped papules or plaques. The lesions progress to ivory-colored atrophic and sclerotic white plaques that may cause phimosis. ${ }^{12}$ LS has specific histologic features, including hyperkeratosis with atrophic epidermis along with the liquefaction degeneration of the basal layer. In the advanced stage, there is a wide homogenous zone extending from the subepidermal layer to the mid-dermis and moderate-to-severe band-like infiltration of chronic inflammatory cells, mainly from $\mathrm{T}$ cells. ${ }^{13,14}$ Our study showed histopathologic evidence of LS in 13 patients (43\%) among the 30 foreskin samples examined, and the incidence was the same as that in the previous reports. ${ }^{10}$ Our study indicated a diagnostic accuracy of 59\% after correlating clinical suspicion of LS and histopathologic diagnosis. Our results differed from those of past reports, ${ }^{5}$ as we overdiagnosed LS. The reason of discrepancy might be that we always perform a physical examination with LS in mind. The pathologic histology of LS ranges from early stage, liquefaction degeneration of the basal cells and a narrow edematous band in the upper dermis, to the late stage, which includes a wide homogenous zone and a band of inflammatory cells in the basal layer. ${ }^{13}$ These differences may lead to clinical discrepancies. These results prove that it is difficult to distinguish LS from phimosis through clinical findings. However, in cases where both whitish and thickened, nonretractable foreskin was observed, our study showed that a histopathologic diagnosis of LS was highly likely.

The purposes of LS treatment are to alleviate voiding problems and prevent anatomical changes such as meatal stenosis and urethral stricture. ${ }^{1}$ Circumcision has been considered to be the standard curative therapy for LS.,4,10 Circumcision is one of the world's most widely performed procedures for religious or cultural reasons; ${ }^{15}$ however, routine neonatal circumcision has never been conducted in Japan. Japanese physicians disagree with circumcision for boys with asymptomatic phimosis, and an absolute indication for circumcision is pathologic phimosis associated with voiding problems or LS. ${ }^{16}$ Becker ${ }^{3}$ reported that partial circumcision is followed by recurrent disease in $50 \%$ of cases. Moreover, topical corticosteroids are the mainstay of treatment for LS at any site in both male and female patients of all ages. Kiss et $\mathrm{al}^{17}$ reported that treatment with topical corticosteroids showed clinical improvement in histologically early- and intermediate-stage LS; however, no improvement was found in the late stages in a double-blind, placebo-controlled, randomized study. Vincent and Mackinnon ${ }^{18}$ also reported that topical, steroid-based cream resolved clinically mild LS 
limited at the prepuce only; however, it was ineffective in cases with established scarring on the foreskin.

Homer et $\mathrm{al}^{19}$ reported that boys with LS were significantly more likely to have a meatus described as abnormal at circumcision (23\%), and they required subsequent meatal procedures, such as meatal dilation and meatotomy, after circumcision in $20 \%$ of cases. On the other hand, only a few boys without LS had an abnormal meatus or required meatal surgery. Additionally, it is important to note that neither an abnormal meatus nor meatal intervention at circumcision significantly influenced the likelihood of undergoing a later meatal operation.

In our study, one patient with histopathologic LS required meatotomy at circumcision, and one patient with histopathologic LS required meatal dilation after circumcision. No patients had urethral strictures.

The limitations of our study include the small number of patients, retrospective data collection, clinical diagnosis of LS based on photography and/or description in the medical record, short observation period, and the possible influence of bias owing to different pathologists. Moreover, the decision to perform surgery was not made because of suspected LS but only for cases of pathologic phimosis associated with voiding problems; recurrent balanoposthitis; or topical steroid treatment resistance. Regarding histopathologic examination, characteristic findings such as liquefaction degeneration and bands of inflammatory cells were observed in cases of LS, although different pathologists diagnosed the patients. Thus, we believe that the histopathologic diagnoses are reliable. In the future, adequately powered prospective trials are required.

\section{Conclusion}

In our study, LS may be diagnosed preoperatively in cases with whitish and thickened, nonretractable foreskins; however, we could not clinically diagnose LS on the basis of preputial appearance alone. Additionally, we could not diagnose LS based on clinical symptoms at all. Our data suggested that it is necessary to analyze the foreskin samples pathologically when LS is suspected clinically. Careful follow-up is needed to detect early complications after surgery in cases with histopathologic LS.

\section{Disclosure}

The authors report no conflicts of interest in this work.

\section{References}

1. Pugliese JM, Morey AF, Peterson AC. Lichen sclerosus: review of the literature and current recommendations for management. $J$ Urol. 2007;178(6):2268-2276.

2. Hayashi Y, Kohri K. Circumcision related to urinary tract infections, sexually transmitted infections, human immunodeficiency virus infections, and penile and cervical cancer. Int J Urol. 2013;20(8):769-775.

3. Becker K. Lichen sclerosus in boys. Dtsch Arztebl Int. 2011;108(4):53-58.

4. Jayakumar S, Antao B, Bevington O, Furness P, Ninan GK. Balanitis xerotica obliterans in children and its incidence under the age of 5 years. J Pediatr Urol. 2012;8(3):272-275.

5. Bochove-Overgaauw DM, Gelders W, de Vylder AM. Routine biopsies in pediatric circumcision: (non) sense? J Pediatr Urol. 2009;5(3): $178-180$.

6. Kreuter A, Kryvosheyeva Y, Terras S, et al. Association of autoimmune diseases with lichen sclerosus in 532 male and female patients. Acto Derm Venereol. 2013;93(2):238-241.

7. Erickson BA, Elliott SP, Myers JB, et al. Understanding the relationship between chronic systemic disease and lichen sclerosus urethral strictures. J Urol. 2016;195(2):363-368.

8. Catterall RD, Oates JK. Treatment of balanitis xerotica obliterans with hydrocortisone injections. Br J Vener Dis. 1962;38:75-77.

9. Shankar KR, Rickwood AM. The incidence of phimosis in boys. BJU Int. 1999;84(1):101-102.

10. Celis S, Reed F, Murphy F, Adams S, Gillick J, Abdelhafeez AH, Lopez PJ. Balanitis xerotica obliterans in children and adolescents: a literature review and clinical series. J Pediatr Urol. 2014;10(1):34-39.

11. Meuli M, Briner J, Hanimann B, Sacher P. Lichen sclerosus et atrophicus causing phimosis in boys: a prospective study with 5-year followup after complete circumcision. J Urol. 1994;152(3):987-989.

12. Depasquale I, Park AJ, Bracka A. The treatment of balanitis xerotica obliterans. BJU Int. 2000;86(4):459-465.

13. Mihara Y, Mihara M, HagariY, Shimao S. Lichen sclerosus et atrophicus. A histological, immunohistochemical and electron microscopic study. Arch Dermatol Res. 1994;286(8):434-442.

14. Hinchliffe SA, Ciftci AO, Khine MM, et al. Composition of the inflammatory infiltrate in pediatric penile lichen sclerosus et atrophicus (balanitis xerotica obliterans): a prospective, comparative immunophenotyping study. Pediatr Pathol. 1994;14(2):223-233.

15. Morris BJ, Wamai RG, Henebeng EB, Tobian AA, Klausner JD, Banerjee J, Hankins CA. Estimation of country-specific and global prevalence of male circumcision. Popul Health Metr. 2016;14:4.

16. Hayashi Y, Kojima Y, Mizuno K, Nakane A, Kamisawa H, Maruyama T, Kohri K. A Japanese view on circumcision: nonoperative management of normal and abnormal prepuce. Urology. 2010;76(1):21-24.

17. Kiss A, Csontai A, Pirót L, Nyirády P, Merksz M, Király L. The response of balanitis xerotica obliterans to local steroid application compared with placebo in children. $J$ Urol. 2001;165(1):219-220.

18. Vincent MV, Mackinnon E. The response of clinical balanitis xerotica obliterans to the application of topical steroid-based creams. J Pediatr Surg. 2005;40(4):709-712.

19. Homer L, Buchanan KJ, Nasr B, Losty PD, Corbett HJ. Meatal stenosis in boys following circumcision for lichen sclerosus (balanitis xerotica obliterans). J Urol. 2014;192(6):1784-1788. 


\section{Publish your work in this journal}

Research and Reports in Urology is an international, peer-reviewed, open access journal publishing original research, reports, editorials, reviews and commentaries on all aspects of adult and pediatric urology in the clinic and laboratory including the following topics: Pathology, pathophysiology of urological disease; Investigation and treatment of urological disease; Pharmacology of drugs used for the treatment of urological disease. The manuscript management system is completely online and includes a very quick and fair peer-review system, which is all easy to use. Visit http://www.dovepress.com/testimonials.php to read real quotes from published authors.

Submit your manuscript here: https://www.dovepress.com/research-and-reports-in-urology-journal 